\title{
Numerical Modeling of Hepatitis B Dynamics with Vertical Transmission and Treatment
}

\section{Razzaq $\mathbf{A}^{1 *}$, Raza $\mathbf{A}^{1}$ and Rafiq $\mathbf{M}^{2}$}

${ }^{1}$ Department of Mathematics, National College of Business Administration \& Economics, Pakistan

${ }^{2}$ Faculty of Engineering, University of Central Punjab, Pakistan

*Corresponding author: Anmole Razzaq, Department of Mathematics National College of Business Administration \& Economics Gujarat, Pakistan, Email: anmolrazzaq709@gmail.com

\section{Research Article}

Volume 3 Issue 3

Received Date: June 25, 2019

Published Date: August 20, 2019

DOI: $10.23880 /$ oajpr-16000181

\section{Abstract}

Numerical modeling of communicable disease is a device to understand the instrument of how disease blowouts and how it can be measured. It builds on our considerate of the spread process of a contagion in a population. In this thesis, we have studied the dynamics of hepatitis B with vertical transmission and treatment numerically. We formulate an unconditionally stable non-standard finite difference (NSFD) scheme for a mathematical model of Hepatitis B disease. The developed numerical scheme is bounded, dynamically consistent and preserves the positivity of the solutions. NSFD scheme shows convergence to the true equilibrium points of the model for any time step sizes. But Euler and RK-4 fail for large time step sizes.

Keywords: Hepatitis B disease; Dynamical system; Numerical modeling; Convergence

Abbreviations: HBV: Hepatitis B Virus; NSFD: NonStandard Finite Difference; DFE: Disease Free Equilibrium; RK-4: Runge-Kutta method of order 4; Differential Equations.

\section{Introduction}

Hepatitis B is a virus who effects the liver [1]. We can say that Hepatitis $B$ is a liver disease that emanates from the infection with Hepatitis B virus (HBV) [2-5]. Its acute infection and chronic infection will be caused [6]. HBV is exposure to infectious blood or body fluids by transmitted. HBV can increase to other humans through the blood:

$>$ HBV arrived the time of birth.

$>$ HBV from contact with other people's blood during childhood.

$>\mathrm{HBV}$ interchange from person to person through blood.

$>\mathrm{HBV}$ transfer child from mother during delivery.
Approximately 2 billion people infected this virus Hepatitis B, 360 million people effected the chronic HBV. 600,000 peoples die in each year with infection of HBV [513].

\section{Mathematical Model}

\section{Variables and Parameters}

$\mathrm{S}_{\mathrm{c}}(\mathrm{t}) \quad$ Denoted by susceptible to chronic infection any time of ' $t$ '.

$\mathrm{U}_{\mathrm{c}}(\mathrm{t}) \quad$ Denoted by chronic infection any time of ${ }^{\prime} \mathrm{t}^{\prime}$.

$I_{c}(t) \quad$ Denoted by chronic infection any time of ' $t$ '.

$\mathrm{S}_{\mathrm{a}}(\mathrm{t}) \quad$ Denoted by susceptible to acute infection any time of ' $t$ '.

$\mathrm{U}_{\mathrm{a}}(\mathrm{t}) \quad$ Denoted by acute infection any time of ' $\mathrm{t}$ '.

$I_{a}(t) \quad$ Denoted by acute infection any time of ' $t$ '.

$\mathrm{T}_{\mathrm{a}}(\mathrm{t}) \quad$ Denoted by treatment who infected by acute virus any time of ' $\mathrm{t}$ '.

$\mathrm{i}=\mathrm{a} \quad$ Denoted by adult females and juveniles. 
NTotal population size.

$\lambda$ Denoted by HBV infection rate.

$\beta$ Denoted by contact the rate with infection individuals.

$\wedge$ Denoted by susceptible adult female's rate.

$\pi$ Denoted by susceptible juvenile's female's rate.

b Denoted by increase susceptible juveniles by birth.

p Denoted by birth from HBV acute females are assumed to susceptible.

$1-\mathrm{p} \quad$ Denoted by remaining birth are infected infants who are in acute virus.

$\gamma_{\mathrm{a}} \quad$ Denoted by acute status progress in chronic virus.

$\gamma_{c} \quad$ Denoted by adult females and juveniles.

$\varepsilon$ Denoted by adult females treated rate. $\eta$ Denoted by adult female's recovery rate and become susceptible.

$\alpha$ Denoted by treated is not perfect and female may progress to chronic stage rate.

$\rho$ Denoted by birth from treated adult females is assumed to be susceptible.

$1-\rho \quad$ Denoted by birth from treated adult females is assumed to be susceptible and remaining proportion is infected and join acute virus.

$\mu$ Denoted by adult female experience natural death.

$\mu_{c} \quad$ Denoted by juvenile's female experience natural death.

$\delta_{\mathrm{a}} \quad$ Denoted by adult female death rate.

$\delta_{\mathrm{c}} \quad$ Denoted by juvenile's female death rate.

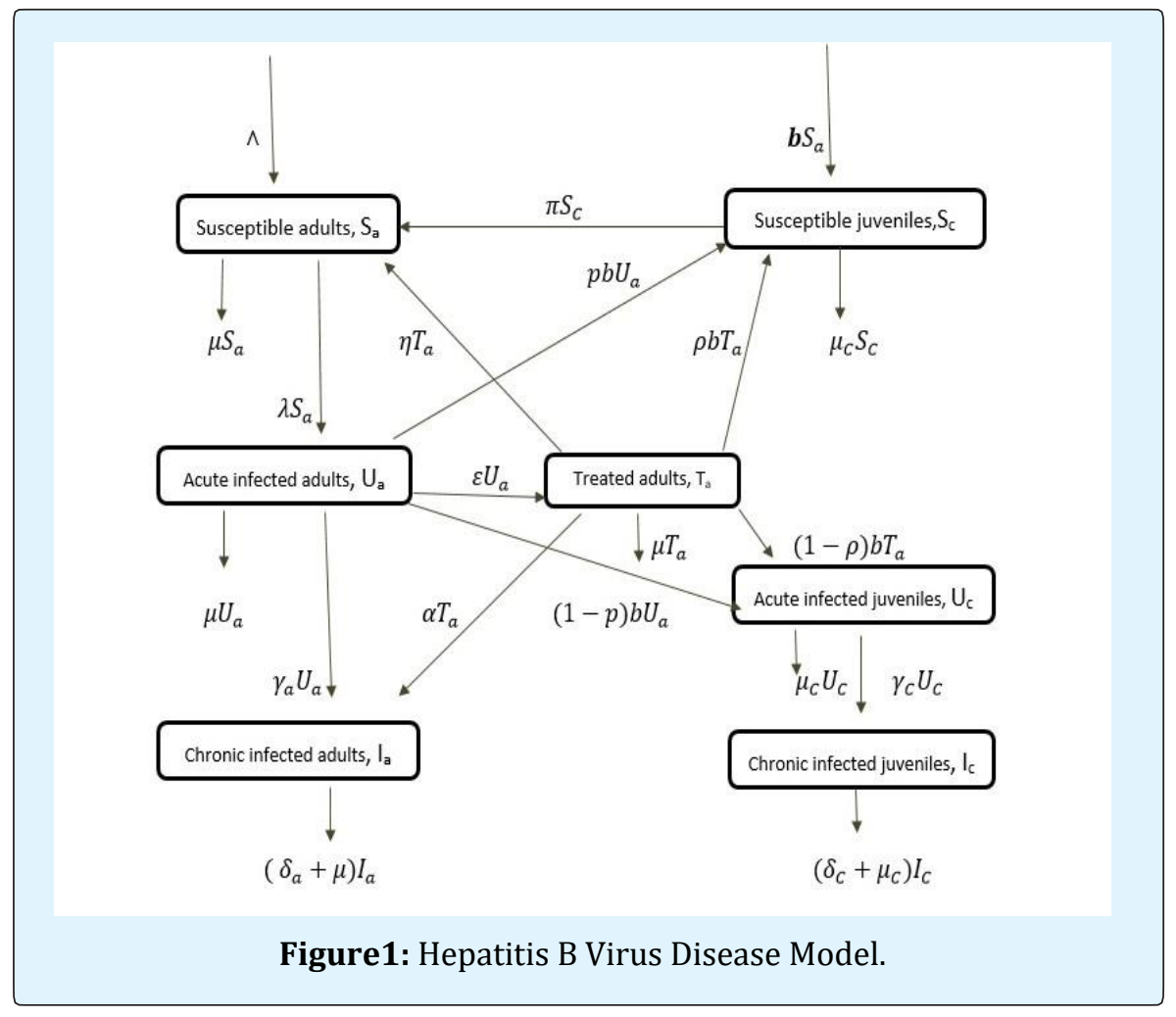

The Scheme of Nonlinear Differential Equations (DE) on behalf of the Typical remains specified by:

$\frac{\mathrm{dS}_{\mathrm{c}}}{\mathrm{dt}}=\mathrm{bS}_{\mathrm{a}}+\mathrm{pbU}_{\mathrm{a}}-\left(\pi+\mu_{\mathrm{c}}\right) \mathrm{S}_{\mathrm{c}}$

$\frac{\mathrm{dU}_{\mathrm{c}}}{\mathrm{dt}}=(1-\mathrm{p}) \mathrm{bU}_{\mathrm{a}}-\left(\mu_{\mathrm{c}}+\gamma_{\mathrm{c}}\right) \mathrm{U}_{\mathrm{c}}$

$\frac{\mathrm{dI}_{\mathrm{c}}}{\mathrm{dt}}=\gamma_{\mathrm{c}} \mathrm{U}_{\mathrm{c}}-\left(\delta_{\mathrm{c}}+\mu_{\mathrm{c}}\right) \mathrm{I}_{\mathrm{c}}$

$\frac{\mathrm{dS}_{\mathrm{a}}}{\mathrm{dt}}=\wedge+\pi \mathrm{S}_{\mathrm{c}}-(\lambda+\mu) \mathrm{S}_{\mathrm{a}}$

$\frac{\mathrm{dU}_{\mathrm{a}}}{\mathrm{dt}}=\lambda \mathrm{S}_{\mathrm{a}}-\left(\mu+\gamma_{\mathrm{a}}+\mathrm{b}\right) \mathrm{U}_{\mathrm{a}}$
$\frac{\mathrm{dI}_{\mathrm{a}}}{\mathrm{dt}}=\gamma_{\mathrm{a}} \mathrm{U}_{\mathrm{a}}-\left(\delta_{\mathrm{a}}+\mu\right) \mathrm{I}_{\mathrm{a}}$

\section{Analysis of the Model}

We describe equilibrium points of system i.e Disease free equilibrium(DFE).

$\mathcal{E}_{1}=\left(\frac{\wedge\left(\pi+\mu_{c}\right)}{\mu\left(\pi+\mu_{c}\right)-\pi b}, \frac{b_{\wedge}}{\mu\left(\pi+\mu_{c}\right)-\pi b}, 0,0,0,0\right)$ are stability facts of scheme (1),

Where $R_{0}=\frac{\beta_{c} \mu\left(\pi+\mu_{c}\right)\left(\mu_{c}+\gamma_{c}\right)\left(1+\theta \gamma_{a}\right)}{\left(\mu\left(\pi+\mu_{c}\right)-\pi b\left(\mu+\gamma_{a}+b\right)\left(\delta_{a}+\mu\right)\right.}$ 


\section{Open Access Journal of Pharmaceutical Research}

$\mathrm{R}_{0}$ recognized as Procreative integer who describes the usual number of inferior impurities introduced of the main impurity. $\mathcal{R}_{0}$ is a beginning influence who describe the disease of the exit or persist? If $\mathcal{R}_{0}<1$ then we say that the scheme will observe disease Free Equilibrium (DFE) and iff $\mathcal{R}_{0}>1$ the scheme to involvement Endemic Equilibrium (EE).

\section{Numerical Modeling}

Now we have conferred two standard finite difference structures to unravel the endless dynamical scheme (1) i.e. Euler's Method and Runge-Kutta Method of Order 4.

\section{Euler Method}

The Forward Euler's Structure for the unceasing model (1) certain through:

$\mathrm{s}_{\mathrm{c}}^{\mathrm{n}+1}-\mathrm{s}_{\mathrm{c}}^{\mathrm{n}}=\mathrm{h}\left\{\mathrm{bs}_{\mathrm{a}}^{\mathrm{n}}+\mathrm{pbU}_{\mathrm{a}}^{\mathrm{n}}-\left(\pi+\mu_{\mathrm{c}}\right) \mathrm{s}_{\mathrm{c}}^{\mathrm{n}}\right\}$

$\mathrm{U}_{\mathrm{c}}^{\mathrm{n}+1}=\mathrm{U}_{\mathrm{c}}^{\mathrm{n}}+\mathrm{h}\left\{(1-\mathrm{p}) \mathrm{b} \mathrm{U}_{\mathrm{a}}^{\mathrm{n}}-\left(\mu_{\mathrm{c}}+\gamma_{\mathrm{c}}\right) \mathrm{U}_{\mathrm{c}}^{\mathrm{n}}\right\}$

$\mathrm{I}_{\mathrm{c}}^{\mathrm{n}+1}=\mathrm{I}_{\mathrm{c}}^{\mathrm{n}}+\mathrm{h}\left\{\gamma_{\mathrm{c}} \mathrm{U}_{\mathrm{c}}^{\mathrm{n}}-\left(\delta_{\mathrm{c}}+\mu_{\mathrm{c}}\right) \mathrm{I}_{\mathrm{c}}^{\mathrm{n}}\right\}$

$\mathrm{s}_{\mathrm{a}}^{\mathrm{n}+1}=\mathrm{s}_{\mathrm{a}}^{\mathrm{n}}+\mathrm{h}\left\{\Lambda+\pi \mathrm{s}_{\mathrm{c}}^{\mathrm{n}}-(\lambda+\mu) \mathrm{s}_{\mathrm{a}}^{\mathrm{n}}\right\}$

$\mathrm{U}_{\mathrm{a}}^{\mathrm{n}+1}=\mathrm{U}_{\mathrm{a}}^{\mathrm{n}}+\mathrm{h}\left\{\lambda s_{\mathrm{a}}^{\mathrm{n}}-\left(\mu+\gamma_{\mathrm{a}}+\mathrm{b}\right) \mathrm{U}_{\mathrm{a}}^{\mathrm{n}}\right\} \mathrm{s}$

$\mathrm{I}_{\mathrm{a}}^{\mathrm{n}+1}=\mathrm{I}_{\mathrm{a}}^{\mathrm{n}}+\mathrm{h}\left\{\gamma_{\mathrm{a}} \mathrm{U}_{\mathrm{a}}^{\mathrm{n}}-\left(\delta_{\mathrm{a}}+\mu\right) \mathrm{I}_{\mathrm{a}}^{\mathrm{n}}\right\}$

\section{Numerical Experiments}

Now solve numerical tryouts by expending the values of given parameters Table 1 [6].

\begin{tabular}{|c|c|}
\hline Parameters & Values \\
\hline$\beta$ & 0.5 \\
\hline$\wedge$ & 0.4 \\
\hline $\mathrm{c}$ & 10.5 \\
\hline $\mathrm{b}$ & 0.8 \\
\hline$\gamma_{\mathrm{a}}$ & 0.7 \\
\hline$\gamma_{\mathrm{c}}$ & 0.054 \\
\hline$\theta$ & 0.88797 \\
\hline $\mathrm{p}$ & 0.6 \\
\hline$\tau$ & 0 \\
\hline$\pi$ & 0.03 \\
\hline$\mu_{\mathrm{c}}$ & 0.4 \\
\hline$\delta_{\mathrm{a}}$ & 0.47 \\
\hline$\delta_{\mathrm{c}}$ & 0.04 \\
\hline$\mu$ & 0.4 \\
\hline$\varepsilon$ & 0 \\
\hline$\eta$ & 0 \\
\hline$\alpha$ & 0 \\
\hline
\end{tabular}

Table 1: Numerical Tryouts.

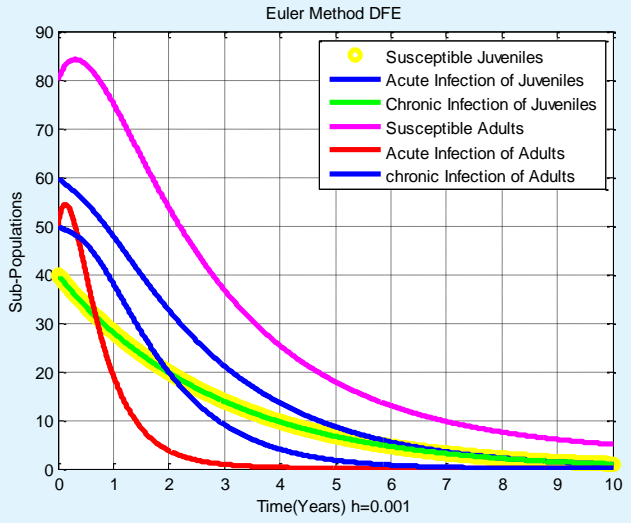

Figure 2: Euler Method (DFE), $\mathrm{h}=0.001$.

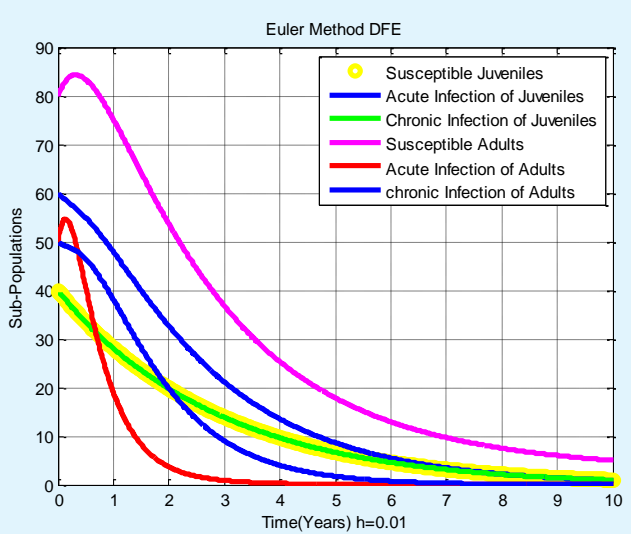

Figure 3: Euler Method (DFE), $\mathrm{h}=0.01$.

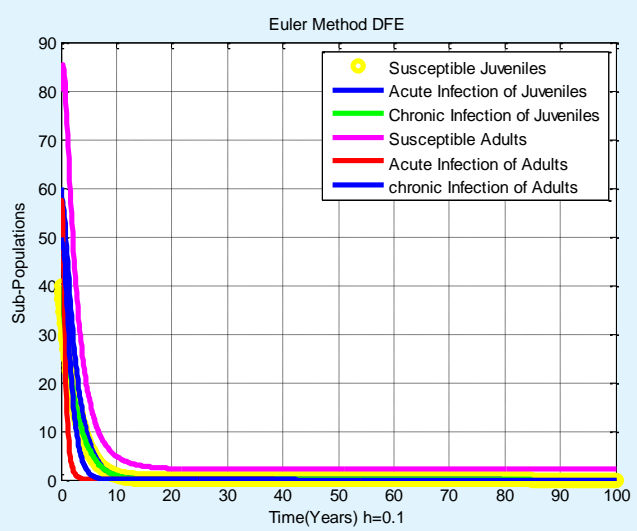

Figure 4: Euler Method (DFE), $\mathrm{h}=0.1$. 


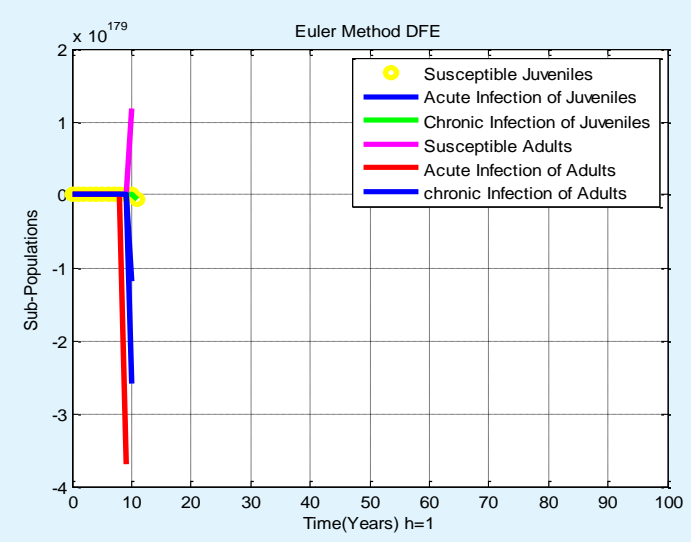

Figure 5: Euler Method (DFE), $\mathrm{h}=1$.

\section{Fourth Order Runge-Kutta Scheme}

For Stage- 1

$\mathrm{K}_{1}=\mathrm{h}\left\{\mathrm{bs}_{\mathrm{a}}^{\mathrm{n}}+\mathrm{pbU}_{\mathrm{a}}^{\mathrm{n}}-\left(\pi+\mu_{\mathrm{c}}\right) \mathrm{s}_{\mathrm{c}}^{\mathrm{n}}\right\}$

$\mathrm{L}_{1}=\mathrm{h}\left\{(1-\mathrm{p}) \mathrm{b} U_{\mathrm{a}}^{\mathrm{n}}-\left(\mu_{\mathrm{c}}+\gamma_{\mathrm{c}}\right) \mathrm{U}_{\mathrm{c}}^{\mathrm{n}}\right\}$

$\mathrm{M}_{1}=\mathrm{h}\left\{\gamma_{\mathrm{c}} \mathrm{U}_{\mathrm{c}}^{\mathrm{n}}-\left(\delta_{\mathrm{c}}+\mu_{\mathrm{c}}\right) \mathrm{I}_{\mathrm{c}}^{\mathrm{n}}\right\}$

$\mathrm{N}_{1}=\mathrm{h}\left\{\Lambda+\pi \mathrm{s}_{\mathrm{c}}^{\mathrm{n}}-(\lambda+\mu) \mathrm{s}_{\mathrm{a}}^{\mathrm{n}}\right\}$

$\mathrm{O}_{1}=\mathrm{h}\left\{\lambda s_{\mathrm{a}}^{\mathrm{n}}-\left(\mu+\gamma_{\mathrm{a}}+\mathrm{b}\right) \mathrm{U}_{\mathrm{a}}^{\mathrm{n}}\right\}$

$\mathrm{P}_{1}=\mathrm{h}\left\{\gamma_{\mathrm{a}} \mathrm{U}_{\mathrm{a}}^{\mathrm{n}}-\left(\delta_{\mathrm{a}}+\mu\right) I_{\mathrm{a}}^{\mathrm{n}}\right\}$

\section{For Stage-2}

$\mathrm{K}_{2}=\mathrm{h}\left[\mathrm{b}\left(\mathrm{s}_{\mathrm{a}}^{\mathrm{n}}+\frac{\mathrm{N}_{1}}{2}\right)+\mathrm{pb}\left(\mathrm{Ua}_{\mathrm{a}}^{\mathrm{n}}+\frac{\mathrm{O}_{1}}{2}\right)-\left(\pi+\mu_{\mathrm{c}}\right)\left(\mathrm{s}_{\mathrm{c}}^{\mathrm{n}}+\frac{\mathrm{K}_{1}}{2}\right)\right]$

$\mathrm{L}_{2}=\mathrm{h}\left[(1-\mathrm{p}) \mathrm{b}\left(\mathrm{U}_{\mathrm{a}}^{\mathrm{n}}+\frac{0_{1}}{2}\right)-\left(\mu_{\mathrm{c}}+\gamma_{\mathrm{c}}\right)\left(\mathrm{U}_{\mathrm{c}}^{\mathrm{n}}+\frac{\mathrm{L}_{1}}{2}\right)\right]$

$\mathrm{M}_{2}=\mathrm{h}\left[\gamma_{\mathrm{c}}\left(\mathrm{U}_{\mathrm{c}}^{\mathrm{n}}+\frac{\mathrm{L}_{1}}{2}\right)-\left(\delta_{\mathrm{c}}+\mu_{\mathrm{c}}\right)\left(\mathrm{I}_{\mathrm{c}}^{\mathrm{n}}+\frac{\mathrm{M}_{1}}{2}\right)\right]$

$\mathrm{N}_{2}=\mathrm{h}\left[\wedge+\pi\left(\mathrm{s}_{\mathrm{c}}^{\mathrm{n}}+\frac{\mathrm{K}_{1}}{2}\right)-(\lambda+\mu)\left(\mathrm{s}_{\mathrm{a}}^{\mathrm{n}}+\frac{\mathrm{N}_{1}}{2}\right)\right]$

$\mathrm{O}_{2}=\mathrm{h}\left[\lambda\left(\mathrm{s}_{\mathrm{a}}^{\mathrm{n}}+\frac{\mathrm{N}_{1}}{2}\right)-\left(\mu+\gamma_{\mathrm{a}}+\mathrm{b}\right)\left(\mathrm{U}_{\mathrm{a}}^{\mathrm{n}}+\frac{\mathrm{o}_{1}}{2}\right)\right]$

$\mathrm{P}_{2}=\mathrm{h}\left[\gamma_{\mathrm{a}}\left(\mathrm{U}_{\mathrm{a}}^{\mathrm{n}}+\frac{\mathrm{O}_{1}}{2}\right)-\left(\delta_{\mathrm{a}}+\mu\right)\left(\mathrm{I}_{\mathrm{a}}^{\mathrm{n}}+\frac{\mathrm{P}_{1}}{2}\right)\right]$

\section{For Stage-3}

$\mathrm{K}_{3}=\mathrm{h}\left[\mathrm{b}\left(s_{\mathrm{a}}^{\mathrm{n}}+\frac{\mathrm{N}_{2}}{2}\right)+\mathrm{pb}\left(\mathrm{U}_{\mathrm{a}}^{\mathrm{n}}+\frac{\mathrm{O}_{2}}{2}\right)-\left(\pi+\mu_{\mathrm{c}}\right)\left(\mathrm{s}_{\mathrm{c}}^{\mathrm{n}}+\frac{\mathrm{K}_{2}}{2}\right)\right]$

$\mathrm{L}_{3}=\mathrm{h}\left[(1-\mathrm{p}) \mathrm{b}\left(\mathrm{U}_{\mathrm{a}}^{\mathrm{n}}+\frac{\mathrm{O}_{2}}{2}\right)-\left(\mu_{\mathrm{c}}+\gamma_{\mathrm{c}}\right)\left(\mathrm{U}_{\mathrm{c}}^{\mathrm{n}}+\frac{\mathrm{L}_{2}}{2}\right)\right]$

$\mathrm{M}_{3}=\mathrm{h}\left[\gamma_{\mathrm{c}}\left(\mathrm{U}_{\mathrm{c}}^{\mathrm{n}}+\frac{\mathrm{L}_{2}}{2}\right)-\left(\delta_{\mathrm{c}}+\mu_{\mathrm{c}}\right)\left(\mathrm{I}_{\mathrm{c}}^{\mathrm{n}}+\frac{\mathrm{M}_{2}}{2}\right)\right]$

$N_{3}=h\left[\wedge+\pi\left(s_{c}^{n}+\frac{K_{2}}{2}\right)-(\lambda+\mu)\left(s_{a}^{n}+\frac{N_{2}}{2}\right)\right]$

$\mathrm{O}_{3}=\mathrm{h}\left[\lambda\left(\mathrm{S}_{\mathrm{a}}^{\mathrm{n}}+\frac{\mathrm{N}_{2}}{2}\right)-\left(\mu+\gamma_{\mathrm{a}}+\mathrm{b}\right)\left(\mathrm{Ua}_{\mathrm{a}}^{\mathrm{n}}+\frac{\mathrm{O}_{2}}{2}\right)\right]$

$\mathrm{P}_{3}=\mathrm{h}\left[\gamma_{\mathrm{a}}\left(\mathrm{U}_{\mathrm{a}}^{\mathrm{n}}+\frac{\mathrm{O}_{2}}{2}\right)-\left(\delta_{\mathrm{a}}+\mu\right)\left(\mathrm{I}_{\mathrm{a}}^{\mathrm{n}}+\frac{\mathrm{P}_{2}}{2}\right)\right]$

\section{For Stage-4}

$\mathrm{K}_{4}=\mathrm{h}\left[\mathrm{b}\left(\mathrm{s}_{\mathrm{a}}^{\mathrm{n}}+\mathrm{N}_{3}\right)+\mathrm{pb}\left(\mathrm{U}_{\mathrm{a}}^{\mathrm{n}}+\mathrm{O}_{3}\right)-\left(\pi+\mu_{\mathrm{c}}\right)\left(\mathrm{s}_{\mathrm{c}}^{\mathrm{n}}+\mathrm{K}_{3}\right)\right]$

$\mathrm{L}_{4}=\mathrm{h}\left[(1-\mathrm{p}) \mathrm{b}\left(\mathrm{U}_{\mathrm{a}}^{\mathrm{n}}+\mathrm{O}_{3}\right)-\left(\mu_{\mathrm{c}}+\gamma_{\mathrm{c}}\right)\left(\mathrm{U}_{\mathrm{c}}^{\mathrm{n}}+\mathrm{L}_{3}\right)\right]$

$\mathrm{M}_{4}=\mathrm{h}\left[\gamma_{\mathrm{c}}\left(\mathrm{U}_{\mathrm{c}}^{\mathrm{n}}+\mathrm{L}_{3}\right)-\left(\delta_{\mathrm{c}}+\mu_{\mathrm{c}}\right)\left(\mathrm{I}_{\mathrm{c}}^{\mathrm{n}}+\mathrm{M}_{3}\right)\right]$

$\mathrm{N}_{4}=\mathrm{h}\left[\Lambda+\pi\left(\mathrm{s}_{\mathrm{c}}^{\mathrm{n}}+\mathrm{K}_{3}\right)-(\lambda+\mu)\left(\mathrm{s}_{\mathrm{a}}^{\mathrm{n}}+\mathrm{N}_{3}\right)\right]$

$\mathrm{O}_{4}=\mathrm{h}\left[\lambda\left(\mathrm{s}_{\mathrm{a}}^{\mathrm{n}}+\mathrm{N}_{3}\right)-\left(\mu+\gamma_{\mathrm{a}}+\mathrm{b}\right)\left(\mathrm{U}_{\mathrm{a}}^{\mathrm{n}}+\mathrm{O}_{3}\right)\right]$

$\mathrm{P}_{4}=\mathrm{h}\left[\gamma_{\mathrm{a}}\left(\mathrm{U}_{\mathrm{a}}^{\mathrm{n}}+\mathrm{O}_{3}\right)-\left(\delta_{\mathrm{a}}+\mu\right)\left(\mathrm{I}_{\mathrm{a}}^{\mathrm{n}}+\mathrm{P}_{3}\right)\right]$

Finally

$s_{c}^{n+1}=s_{c}^{n}+\frac{1}{6}\left[K_{1}+2 K_{2}+2 K_{3}+K_{4}\right]$

$$
\begin{aligned}
& \mathrm{U}_{\mathrm{c}}^{\mathrm{n}+1}=\mathrm{U}_{\mathrm{c}}^{\mathrm{n}}+\frac{1}{6}\left[\mathrm{~L}_{1}+2 \mathrm{~L}_{2}+2 \mathrm{~L}_{3}+\mathrm{L}_{4}\right] \\
& \mathrm{I}_{\mathrm{c}}^{\mathrm{n}+1}=\mathrm{I}_{\mathrm{c}}^{\mathrm{n}}+\frac{1}{6}\left[\mathrm{M}_{1}+2 \mathrm{M}_{2}+2 \mathrm{M}_{3}+\mathrm{M}_{4}\right] \\
& \mathrm{S}_{\mathrm{a}}^{\mathrm{n}+1}=\mathrm{S}_{\mathrm{a}}^{\mathrm{n}}+\frac{1}{6}\left[\mathrm{~N}_{1}+2 \mathrm{~N}_{2}+2 \mathrm{~N}_{3}+\mathrm{N}_{4}\right] \\
& \mathrm{U}_{\mathrm{a}}^{\mathrm{n}+1}=\mathrm{U}_{\mathrm{a}}^{\mathrm{n}}+\frac{1}{6}\left[\mathrm{O}_{1}+2 \mathrm{O}_{2}+2 \mathrm{O}_{3}+\mathrm{O}_{4}\right] \\
& \mathrm{I}_{\mathrm{a}}^{\mathrm{n}+1}=\mathrm{I}_{\mathrm{a}}^{\mathrm{n}}+\frac{1}{6}\left[\mathrm{P}_{1}+2 \mathrm{P}_{2}+2 \mathrm{P}_{3}+\mathrm{P}_{4}\right]
\end{aligned}
$$

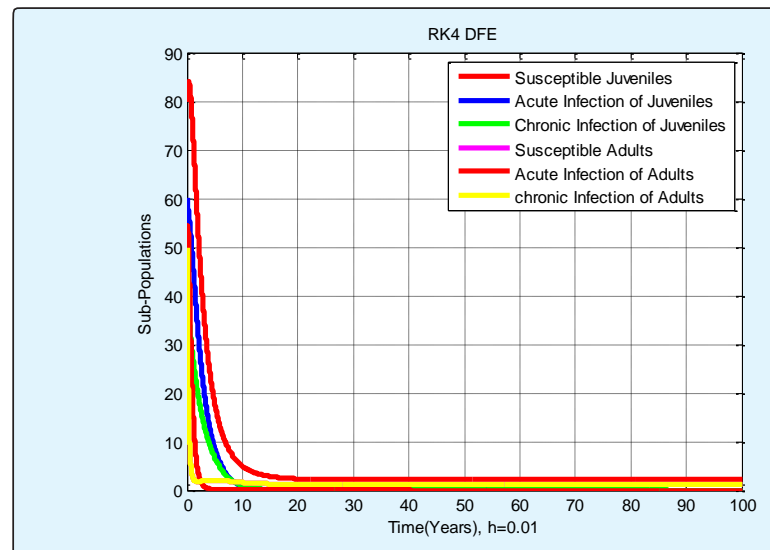

Figure 6: RK-4 Method (DFE), $\mathrm{h}=0.01$.

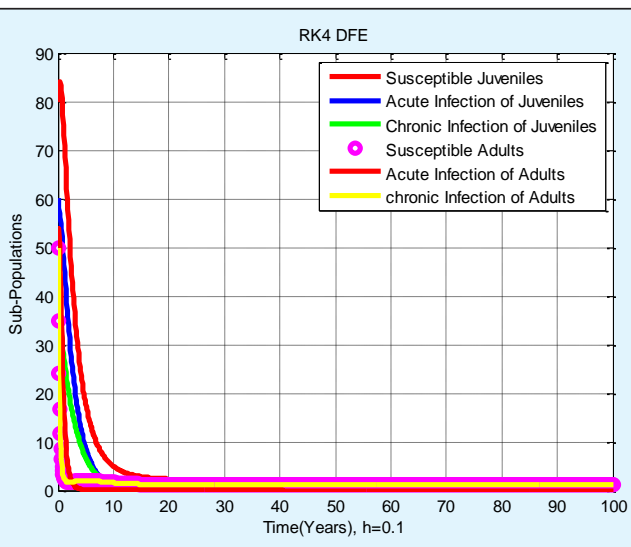

Figure 7: RK-4 Method (DFE), $\mathrm{h}=0.1$.

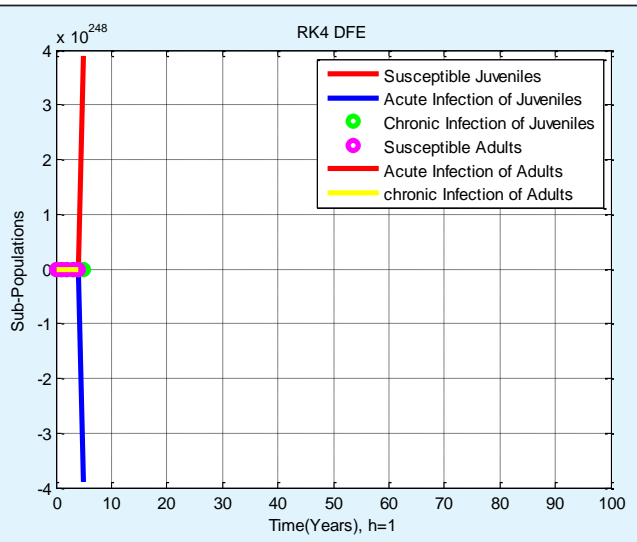

Figure 8: RK-4 Method (DFE), $\mathrm{h}=1$. 


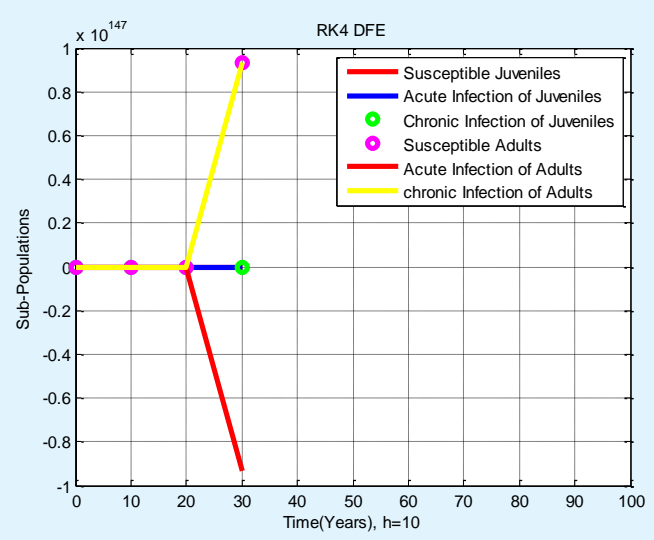

Figure 9: RK-4 Method (DFE), $\mathrm{h}=10$.

\section{Non-Standard Finite Difference Model}

Now we show an unreservedly convergent nonstandard finite difference(NSFD) numerical model which be there describe on non-standard finite difference modeling concept introduced by Micken's [3]. Now show the covergenence scrutiny of the suggested structure.The NSFD model for the incessant dynamical system is given by:

$$
\begin{aligned}
\mathrm{s}_{\mathrm{c}}^{\mathrm{n}+1}= & \frac{\mathrm{s}_{\mathrm{c}}^{\mathrm{n}}+\mathrm{h}\left(\mathrm{b} s_{\mathrm{a}}^{\mathrm{n}}+\mathrm{pbU} \mathrm{U}_{\mathrm{a}}^{\mathrm{n}}\right)}{\left(1+\mathrm{h}\left(\pi+\mu_{\mathrm{c}}\right)\right)} \\
\mathrm{U}_{\mathrm{c}}^{\mathrm{n}+1} & =\frac{\mathrm{U}_{\mathrm{c}}^{\mathrm{n}}+\mathrm{h}(1-\mathrm{p}) \mathrm{b} \mathrm{U}_{\mathrm{a}}^{\mathrm{n}}}{\left(1+\mathrm{h}\left(\mu_{\mathrm{c}}+\gamma_{\mathrm{c}}\right)\right)} \\
\mathrm{I}_{\mathrm{c}}^{\mathrm{n}+1} & =\frac{\mathrm{I}_{\mathrm{c}}^{\mathrm{n}}+\mathrm{h} \gamma_{\mathrm{c}} \mathrm{U}_{\mathrm{c}}^{\mathrm{n}}}{\left(1+\mathrm{h}\left(\delta_{\mathrm{c}}+\mu_{\mathrm{c}}\right)\right)} \\
\mathrm{S}_{\mathrm{a}}^{\mathrm{n}+1} & =\frac{\mathrm{s}_{\mathrm{a}}^{\mathrm{n}}+\mathrm{h}\left(\Lambda+\pi s_{c}^{\mathrm{n}}\right)}{(1+\mathrm{h}(\lambda+\mu))} \\
\mathrm{U}_{\mathrm{a}}^{\mathrm{n}+1} & =\frac{\mathrm{U}_{\mathrm{a}}^{\mathrm{n}}+\mathrm{h} \lambda s_{\mathrm{a}}^{\mathrm{n}}}{\left(1+\mathrm{h}\left(\mu+\gamma_{\mathrm{a}}+\mathrm{b}\right)\right)} \\
\mathrm{I}_{\mathrm{a}}^{\mathrm{n}+1} & =\frac{\mathrm{I}_{\mathrm{a}}^{\mathrm{n}}+\mathrm{h} \gamma_{\mathrm{a}} U_{\mathrm{a}}^{\mathrm{n}}}{\left(1+\mathrm{h}\left(\delta_{\mathrm{a}}+\mu\right)\right)}
\end{aligned}
$$

\section{Convergence Analysis of NSFD Scheme}

Let us define

$$
\begin{aligned}
& \mathrm{F}=\frac{\mathrm{s}_{\mathrm{c}}^{\mathrm{n}}+\mathrm{h}\left(\mathrm{b} \mathrm{s}_{\mathrm{a}}^{\mathrm{n}}+\mathrm{pbU_{a } ^ { \mathrm { n } }}\right)}{\left(1+\mathrm{h}\left(\pi+\mu_{\mathrm{c}}\right)\right)} \\
& \mathrm{G}=\frac{\mathrm{U}_{\mathrm{c}}^{\mathrm{n}}+\mathrm{h}(1-\mathrm{p}) \mathrm{b} U_{\mathrm{a}}^{\mathrm{n}}}{\left(1+\mathrm{h}\left(\mu_{\mathrm{c}}+\gamma_{\mathrm{c}}\right)\right)} \\
& \mathrm{H}=\frac{\mathrm{I}_{\mathrm{c}}^{\mathrm{n}}+\mathrm{h} \gamma_{\mathrm{c}} \mathrm{U}_{\mathrm{c}}^{\mathrm{n}}}{\left(1+\mathrm{h}\left(\delta_{\mathrm{c}}+\mu_{\mathrm{c}}\right)\right)} \\
& I=\frac{s_{a}^{n}+h\left(\wedge+\pi s_{c}^{n}\right)}{(1+h(\lambda+\mu))} \\
& J=\frac{U_{a}^{n}+h \lambda s_{a}^{n}}{\left(1+h\left(\mu+\gamma_{a}+b\right)\right)} \\
& \mathrm{K}=\frac{\mathrm{I}_{\mathrm{a}}^{\mathrm{n}}+\mathrm{h} \gamma_{\mathrm{a}} \mathrm{U}_{\mathrm{a}}^{\mathrm{n}}}{\left(1+\mathrm{h}\left(\delta_{\mathrm{a}}+\mu\right)\right)}
\end{aligned}
$$

Now the Jacobian Matrix is given by

$$
\mathrm{J}=\left[\begin{array}{llllll}
\frac{\partial \mathrm{F}}{\partial \mathrm{S}_{\mathrm{c}}} & \frac{\partial \mathrm{F}}{\partial \mathrm{U}_{\mathrm{c}}} & \frac{\partial \mathrm{F}}{\partial \mathrm{I}_{\mathrm{c}}} & \frac{\partial \mathrm{F}}{\partial \mathrm{S}_{\mathrm{a}}} & \frac{\partial \mathrm{F}}{\partial \mathrm{U}_{\mathrm{a}}} & \frac{\partial \mathrm{F}}{\partial \mathrm{I}_{\mathrm{a}}} \\
\frac{\partial \mathrm{G}}{\partial \mathrm{S}_{\mathrm{c}}} & \frac{\partial \mathrm{G}}{\partial \mathrm{U}_{\mathrm{c}}} & \frac{\partial \mathrm{G}}{\partial \mathrm{I}_{\mathrm{c}}} & \frac{\partial \mathrm{G}}{\partial \mathrm{S}_{\mathrm{a}}} & \frac{\partial \mathrm{G}}{\partial \mathrm{U}_{\mathrm{a}}} & \frac{\partial \mathrm{G}}{\partial \mathrm{I}_{\mathrm{a}}} \\
\frac{\partial \mathrm{H}}{\partial \mathrm{S}_{\mathrm{c}}} & \frac{\partial \mathrm{H}}{\partial \mathrm{U}_{\mathrm{c}}} & \frac{\partial \mathrm{H}}{\partial \mathrm{I}_{\mathrm{c}}} & \frac{\partial \mathrm{H}}{\partial \mathrm{S}_{\mathrm{a}}} & \frac{\partial \mathrm{H}}{\partial \mathrm{U}_{\mathrm{a}}} & \frac{\partial \mathrm{H}}{\partial \mathrm{I}_{\mathrm{a}}} \\
\frac{\partial \mathrm{I}}{\partial \mathrm{S}_{\mathrm{c}}} & \frac{\partial \mathrm{I}}{\partial \mathrm{U}_{\mathrm{c}}} & \frac{\partial \mathrm{I}}{\partial \mathrm{I}_{\mathrm{c}}} & \frac{\partial \mathrm{I}}{\partial \mathrm{S}_{\mathrm{a}}} & \frac{\partial \mathrm{I}}{\partial \mathrm{U}_{\mathrm{a}}} & \frac{\partial \mathrm{I}}{\partial \mathrm{I}_{\mathrm{a}}} \\
\frac{\partial \mathrm{J}}{\partial \mathrm{S}_{\mathrm{c}}} & \frac{\partial \mathrm{J}}{\partial \mathrm{U}_{\mathrm{c}}} & \frac{\partial \mathrm{J}}{\partial \mathrm{I}_{\mathrm{c}}} & \frac{\partial \mathrm{J}}{\partial \mathrm{S}_{\mathrm{a}}} & \frac{\partial \mathrm{J}}{\partial \mathrm{U}_{\mathrm{a}}} & \frac{\partial \mathrm{J}}{\partial \mathrm{I}_{\mathrm{a}}} \\
\frac{\partial \mathrm{K}}{\partial \mathrm{S}_{\mathrm{c}}} & \frac{\partial \mathrm{K}}{\partial \mathrm{U}_{\mathrm{c}}} & \frac{\partial \mathrm{K}}{\partial \mathrm{I}_{\mathrm{c}}} & \frac{\partial \mathrm{K}}{\partial \mathrm{S}_{\mathrm{a}}} & \frac{\partial \mathrm{K}}{\partial \mathrm{U}_{\mathrm{a}}} & \frac{\partial \mathrm{K}}{\partial \mathrm{I}_{\mathrm{a}}}
\end{array}\right]
$$

At DiseaseFree Equilibrium

$\mathcal{E}_{1}=\left(\frac{\wedge\left(\pi+\mu_{c}\right)}{\mu\left(\pi+\mu_{c}\right)-b \pi}, \frac{b \wedge}{\mu\left(\pi+\mu_{c}\right)-b \pi}, 0,0,0,0\right)$

There are the following eigen values of above jacobian matrix is:

$$
\begin{aligned}
\Rightarrow & \lambda_{1}=\frac{1}{\left(1+\mathrm{h}\left(\pi+\mu_{\mathrm{c}}\right)\right)}<1 \\
\Rightarrow & \lambda_{2}=\frac{1}{\left(1+\mathrm{h}\left(\mu_{\mathrm{c}}+\gamma_{\mathrm{c}}\right)\right)}<1 \\
\Rightarrow & \lambda_{3}=\frac{1}{\left(1+\mathrm{h}\left(\delta_{\mathrm{c}}+\mu_{\mathrm{c}}\right)\right)}<1 \\
\Rightarrow & \lambda_{4}=\frac{1}{(1+\mathrm{h}(\lambda+\mu))}<1 \\
\Rightarrow & \lambda_{5}=\frac{1}{\left(1+\mathrm{h}\left(\mu+\gamma_{\mathrm{a}}+\mathrm{b}\right)\right)}<1 \\
\Rightarrow & \lambda_{6}=\frac{1}{\left(1+\mathrm{h}\left(\delta_{\mathrm{a}}+\mu\right)\right)}<1
\end{aligned}
$$

S0,

The scheme is LAS.

\section{Numerical Experiments}

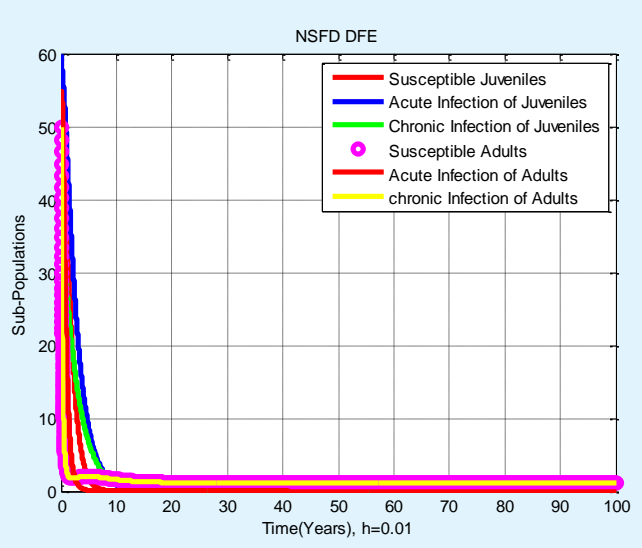

Figure 10: NSFD Method (DFE), $\mathrm{h}=0.01$. 


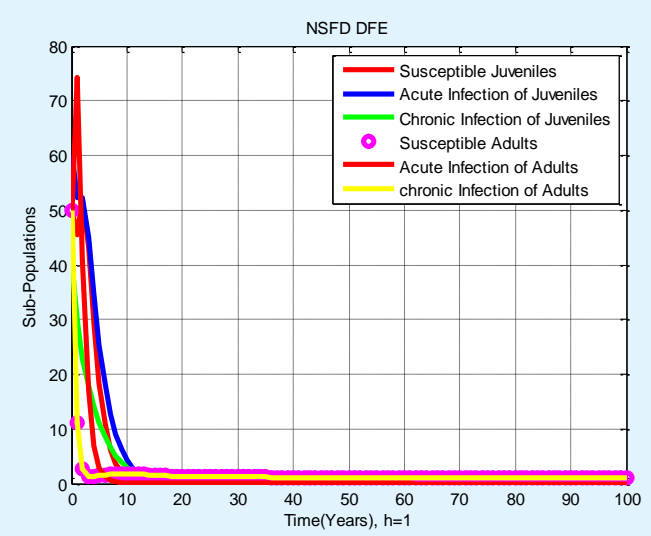

Figure 11: NSFD Method (DFE), $\mathrm{h}=1$.

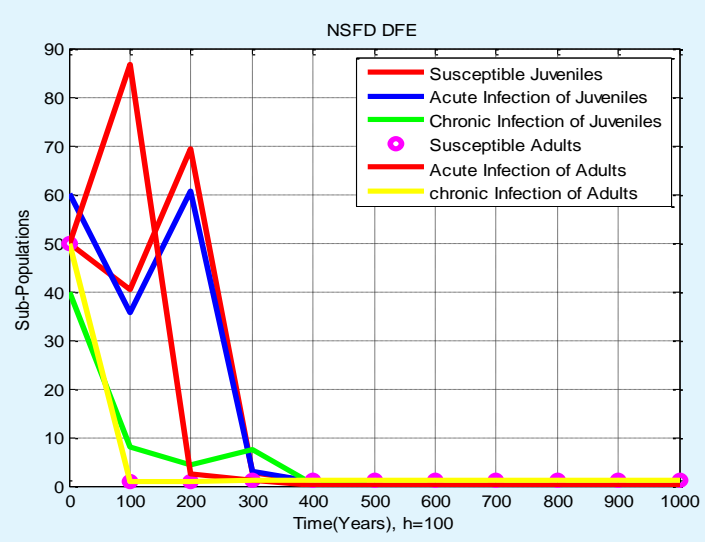

Figure 12: NSFD Method (DFE), $\mathrm{h}=100$.

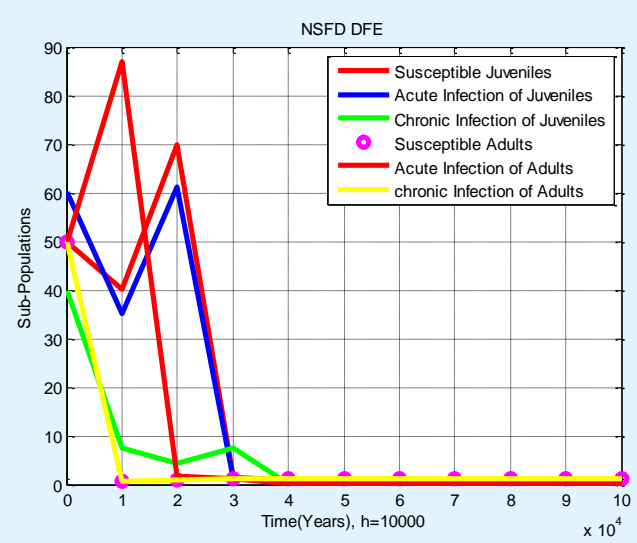

Figure 13: NSFD Method (DFE), $h=10000$.

\section{Comparison Analysis}

In this section, we see the comparison among of two standard difference schemes and non-standard difference scheme in epidemiology

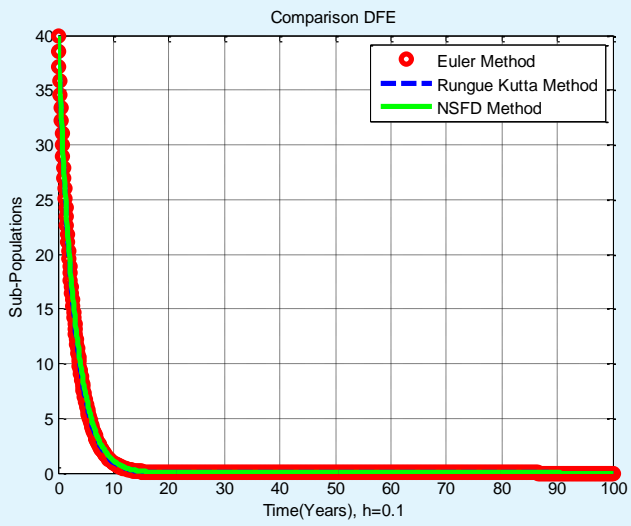

Figure 14: Comparison (DFE), $\mathrm{h}=0.1$.

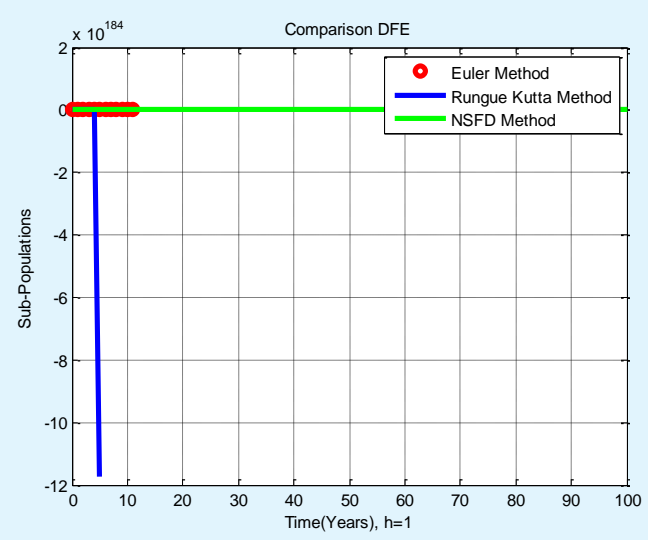

Figure 15: Comparison (DFE), $\mathrm{h}=1$.

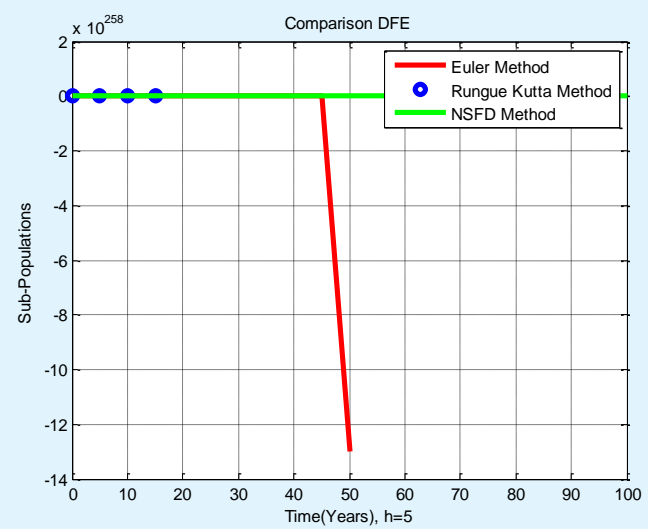

Figure 16: Comparison (DFE), $h=5$. 


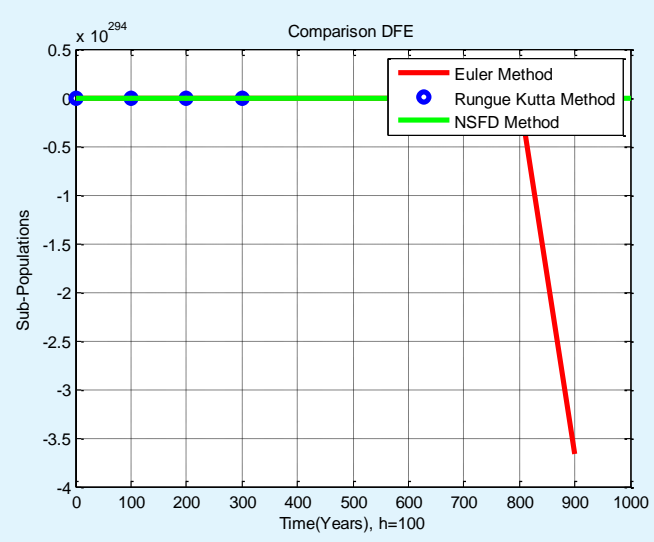

Figure 17: Comparison (DFE), $\mathrm{h}=100$.

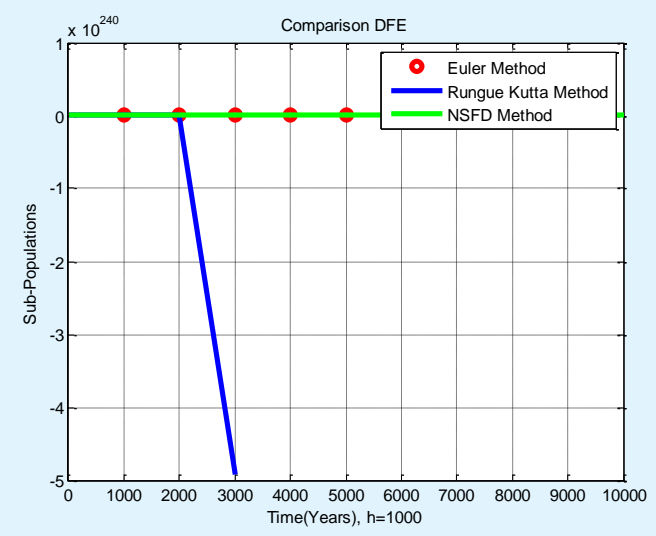

Figure 18: Comparison (DFE), $h=1000$.

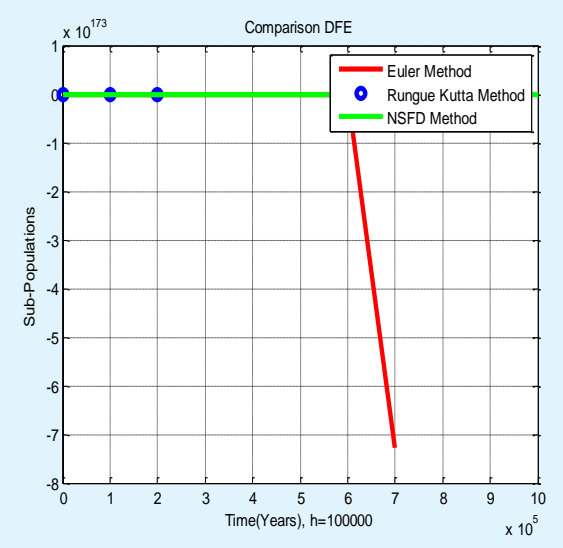

Figure 19: Comparison (EE), $h=100000$.

\section{Results and Discussion}

The model of transmission dynamics of Hepatitis B virus disease consumes introduced expending PSIT Model. (i.e Threatened, Susceptible, Infected and Treated). The constancy of solid positions i.e the Disease free equilibrium(DFE) deliberated numerically. We describe an unqualifiedly constant Non-Standard Finite Difference (NSFD) structure aimed at the incessant dynamical system. The suggested structure exists dynamical consistant, numerically steady and holds all the athentic assets of the incessant model. The outcomes equaled well known standard finite difference schemes i.e Euler's and Runge-Kutta method of order 4 (RK-4). The Euler and RK-4 are provisionally convergent and diverge of the assured ethics of step size ' $h$ ' while the constructed NSFD scheme for every assessment used to residues convergent [14-20].

\section{Conclusion}

The non - standard finite difference scheme created for the communication dynamics of HBV is unconditionally convergent. Inappropriately the abovementioned schemes like Euler and RK-4 are unsuccessful they depend on step size. So, Euler and RK-4 are conditionally convergent. When we intensify the step size, the graph of Euler and RK-4 are divergent and from time to time give variation in solution. The new advanced numerical scheme like non-standard finite difference scheme is independent on step size. Uncertainty we intensify the step size in hundreds and thousands then NSFD motionless convergent. The NSFD scheme is informal implement that gives mathematically stable, positivity, bounded-ness and shows an equal behaviour of the continuous model and discrete model.

\section{References}

1. Shaban N, Manoza D (2016) Hepatitis B Virus Disease: A mathematical model for vertical transmission with treatment strategy. Mathematical Theory and Modeling 6(6): 25-35.

2. Sirilert S, Traisrislip K, Siritanapa $\mathrm{P}$, Tongsong $\mathrm{T}$ (2014) Pregnancy outcomes among chronic carrier of hepatitis b virus. Inte J Gynecol Obstet 126(2): 106110.

3. Gentile I, Borgia G (2014) Vertical transmission of hepatitis $\mathrm{b}$ virus: challenges and solutions. Inte J Womens Health 6: 605-611. 


\section{Open Access Journal of Pharmaceutical Research}

4. Kamyad VA, Akbari R, Heydari AA, Heydari A (2014) Mathematical modeling of transmission dynamics and optimal control of vaccination treatment for hepatitis $B$ virus". Computational and mathematical methods in medicine pp: 1-15.

5. Borgia G, Calrleo MA, Gaeta GB, GentileI (2012) Hepatitis B in pragancy. World J Gastroenterol 18(34): 4677-4683.

6. WHO (2012) Prevention and control of viral hepatitis infection. Framework for Global Action pp: 1-28.

7. Zoul L, Zhang W, Ruan S, (2010) Modeling the transmission dynamics and control of hepatitis B virus in China. J Theor Biol 262: 330-338.

8. Jonas MM (2009) Hepatitis B and pregnancy: an underestimated issue. Liver Int 29(1): 133-139.

9. Chitnis N, Hyman JM, Cushing JM (2008) Determining important parameters in the spread of malaria through the sensitivity analysis of a mathematical model. Bull Math Biol 70(5): 1272-1296.

10. Ciupe SM, Ribeiro RM, Nelson PW (2007) Modelling of acute hepatitis b virus infected. J Theor Biol 247(1): 23-35.

11. Shepard CW, Simard EP, Finelli L, Fiore AE, Bell BP (2006) Hepatitis B virus infection: epidemiology and vaccination. Epidemiol Rev 28: 112-125.

12. Goldstein ST, Zhou F, Hadler SC, Bell BP, Mast EE, et al. (2005) A mathematical model to estimate global hepatitis B disease burden and vaccination impact. Inte J Epidemiol 34(6): 1329-1339.
13. Lavanchy D (2004) Hepatitis B virus epidemiology, disease burden, treatment and current and emerging prevention and control measures. J Viral Hepat 11(2): 97-107.

14. Gumel AB, Moghadas SM (2003) Qualitative study of vaccination model with a non-linear incidence. Applied mathematics and computational 143(2-3): 403-419.

15. Van den Driessche P, Watmough J (2002) Reproduction number and Sub-threshold endemic equilibria for compartmental models of disease transmission. Mathematical Biosciences 180(1-2): 2948.

16. Hethcote HW (2000) The mathematics of infectious diseases. SIAM Rev 42(4): 599-653.

17. Hyams KC (1995) Risk of chronicity following acute hepatitis B virus infection: a review. Clin Infect Dis 20(4): 992-1000.

18. Edmunds WJ, Medley GF, Nokes DJ, Hall AJ, Whittle HC (1993) The influence of age on the development of the hepatitis b carrier state. Proc BioSci 253(1337): 197-201.

19. Diekmann O, Heestebeek JA, Metz JAJ (1990) On the definition and the computation of the basic Reproduction Ratio, $\mathrm{R}_{0}$ in models of infectious Disease in Heterogeneous Population. J Math Bio 28(4): 365382.

20. Lakshmikanthan V, Bainov D, Simeonov PS (1989) Theory of mpulsive differential equation. World scientific singapore. 